\title{
Energy Harvesting for Wireless Electric power Transmission Based on Analysing Inductive coupling
}

\author{
ZHAO Guo Zhu1, a, LI Zheng Quan ${ }^{1, b}$, CHEN You Jun ${ }^{1, c}$ \\ ${ }^{1}$ Civil Aviation Flight University of China Guanghan Sichuan, China \\ ajude.1909@163.com, b379175094@qq.com, c371443975@qq.com
}

Keywords: wireless electric power transmission, Inductive coupling, nonresonant induction.

Abstract. With parameter values chosen to obtain good calculate, the experimental results show that the load will receive the maximum voltage and maximum electric power at resonant frequent $22 \mathrm{kHz}$ when Tesla coils are $4 \mathrm{~mm}$ away. The maximum electric power transfered by two Tesla coils of diameter $30 \mathrm{~mm}$ with 13 loops per Tesla coil is at $1.4 \mathrm{~W}$ when the distance separating the Tesla coils is $4 \mathrm{~mm}$. Under the resonance frequency, about $42 \%$ of the electric power is delivered to the load that is supplied by a sinusoidal AC source (electric power function generator).

\section{Introduction}

The ability to provide electric power for these and other electric devices wirelessly would greatly increase their portability and accessibility for the public. The past decade has witnessed a surge in the use of wireless electric power transmission. As a consequence, profit in transmission via Inductive coupling has reemerged. Radiative Transmission, although perfectly suitable for transfer information, poses a number of difficulties for electric power Transmission applications: The efficiency of electric power Transmission is still low ${ }^{[1]}$.

Inductive coupling is an old and well-understood method of wireless electric power Transmission. The source drives a primary Tesla coil, creating a sinusoidally varying magnetic field, which induces a voltage across the terminals of a secondary Tesla coil, and thus Transmission electric power to a load ${ }^{[2,3,4]}$.

Research has been done which indicated that electric power Transmission can be achieved efficiently using two or more resonant objects of the same resonance frequency. As shown in figure 1, a schematic electric circuit of electromagnetic induction which consist of a source Tesla coil pair and a single receiving Tesla coil pair. Single receiving Tesla coil pair consists of $L_{2}, L_{1}$. Below is an analysis of how magnetic induction generates electric power.

\section{Resonance Frequency}

The energy is transferred in form of electromagnetic wave, where ${ }^{[4,5,6]}$ :

$$
\begin{aligned}
& H=\frac{B}{\mu_{0}}=x I, \text { where } x=\frac{r^{2}}{2\left(r^{2}+D^{2}\right)} \\
& U=\frac{E B}{\mu_{0}}=c \mu_{0} H^{2}, \text { where } E=c B
\end{aligned}
$$

$H$-Magnetic field intensity; $B$-Magnetic flux; $U$-Energy; $E$-Electric field; $r$ : radius of the loop; $D$ - distance between the Tesla coils; $k$ - coupling ratio. The magnetic field from a loop is given by

$$
\begin{aligned}
& H=K \cdot I\left(r^{2} / 2\left(r^{2}+D^{2}\right)^{3 / 2}\right) \cdot e^{-\gamma D} \\
& I(z)=2 r \cdot H(z)
\end{aligned}
$$

and $\gamma$ is the loss in the medium, where:

$$
\gamma=\sqrt{-1.1126 \times 10^{-17} \omega^{2}+3.33 \times 10^{-9} j \omega}
$$


The new and effective technique for increasing the energy received by the Tesla coil is to add a parallel or serial capacitor to the secondary side to form a resonant electric circuit under the different frequency ${ }^{[7,8]}$. Figure 2 show a schematic electric circuit drawing of Inductive coupling. An inductive electric power Transmission model is built to show correlation theory. An $A C$ source drives a primary Tesla coil pair and a secondary receiving Tesla coil pair is close to primary Tesla coil. The secondary receiving Tesla coil pair consists of $L_{2}, R_{2}, C_{2}, R_{L}$. In the system of figure 2 , the $A C$ source drives multiturn Tesla coil $L_{1}$ that is inductively coupled to another multiturn Tesla coil $L_{2}$ of the same diameter. The resistances $\mathrm{R}_{1}$ and $\mathrm{R}_{2}$ are the resistances of the Tesla coils, while $R_{S}$ is the internal resistance of the source, and $\mathrm{R}_{\mathrm{L}}$ is the load resistance. The $R_{L}$ in Figure 2 and other figure are linear elements, used to demonstrate wireless electric power Transmission's effect. This electric circuit representation described here and in subsequent sections use a bulb as resistive load ${ }^{[9]}$.

\section{Schematic Electric Circuit Drawing of Inductive Coupling}

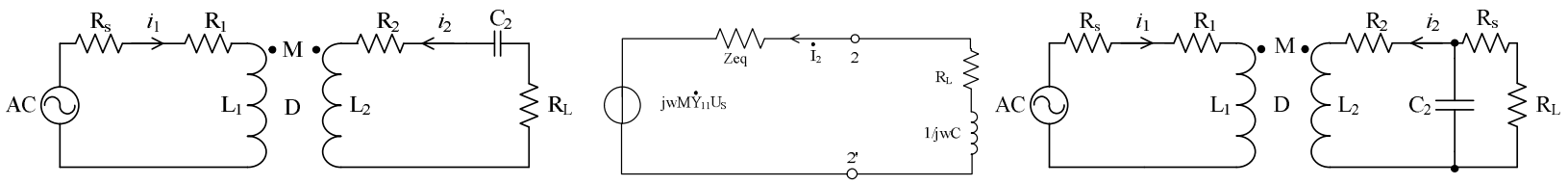

Figure 1.A schematic electric circuit drawing of Inductive coupling

Figure 2. Equivalent electric circuit (primary side convert to secondary side)

Figure 3. A schematic electric circuit of a source Tesla coil pair and a receiving one.

Under the above conditions, parallel resonant can occur, at last reach wireless electric power transmission based on resonance. Figure 2 shows electric circuit model, after an electrical inductance $L_{2}$ and a capacitance $C_{2}$ are resonant, the resonant between $L_{2} C_{2}$ and source will occur. The resonant frequency is given by:

$$
f_{0}=1 / 2 \pi \sqrt{L C}
$$

The impedance of this electric circuit is:

$$
Z=R_{L}+j(w L-1 / w C)
$$

At resonant frequency, $w L=1 / w C, w^{2}=1 / L C, f_{S}=1 / 2 \pi(\mathrm{LC})^{1 / 2}$. This formula is used to solve frequency matching problem. As a result, the electric power consumed in the parasitic impedances is very little and can be negligible. In this electric circuit, a serial electric circuit is designed and parallel electric circuit will be studied in the later. To find the value of the capacitor, let $L_{l}=30.9 \mu \mathrm{H}$. The Figure 1 can be modeled at $f=1 \mathrm{kHz} \sim 30 \mathrm{kHz}$. The figure above gives $L_{2}=31.2 \mu \mathrm{H}$. $L_{1}$ and $L_{2}$ are the values of primary Tesla coil and secondary Tesla coil. The two Tesla coils are made of electrically conducting wire and they are approximately same. The distance between $L_{1}$ and $L_{2}$ is labeled $D^{[10]}$.

In the system of figure 3 , the following linear equations are obtained ${ }^{[3,6]}$.

$$
\begin{aligned}
& X_{L}=X_{C} \\
& Q=\frac{\rho}{Z_{0}}=\frac{\omega_{0} L}{R}=\frac{1}{\omega_{0} C R}=\frac{\sqrt{L / C}}{R} \\
& |Z|=\left|R+j\left(X_{L}-X_{C}\right)\right|=\sqrt{R^{2}+\left(X_{L}-X_{C}\right)^{2}} \\
& k=\frac{M}{\sqrt{L_{1} L_{2}}} \\
& L_{e q}=\frac{\left(L_{1} L_{2}-M^{2}\right)}{L_{1}+L_{2}+2 M} \quad \geq 0 \\
& R_{r}=20(2 \pi / \lambda)^{4}\left(\pi a^{2} n\right)^{2}
\end{aligned}
$$$$
\text { (8) } \rho=\omega_{0} L=\frac{1}{\omega_{0} C}=\sqrt{\frac{L}{C}}
$$$$
\text { (10) } \left.\left.U_{L}=I_{0} X_{L}=U_{C}=I_{0} X_{C}\right\rangle\right\rangle U=I_{0} R
$$$$
\text { (12) } Z=R+j\left(\omega L-\frac{1}{\omega C}\right)
$$$$
\text { (14) } \quad k \stackrel{\text { def }}{=} \frac{M}{\sqrt{L_{1} L_{2}}} \leq 1
$$$$
\text { (16) } Z_{\text {eq }}=R_{2}+j w L_{2}+(w M)^{2} Y_{11}
$$

Where, $R_{r}$-radiation resistance; $n$-Tesla coil turn; $R$-radius of Tesla coil $(\mathrm{m}) ; \lambda$-wave length, $\lambda=c / f, c=3.00 \times 10^{8} ; a$-radius of conductor section $(\mathrm{m})$. 
Tesla coil 2, then has the largest radiation resistance, which is far small in comparison with the resistance RL. So $\mathrm{Rr}$ is to be neglected. It is easy to show that the key to efficient of energy Transmission is $Z_{e q}=j X_{c}$.

Paper [1] and [9] present a coupled electric circuit mode which works depend on two resonant Tesla coils mainly. A $L C$ resonant electric circuit under the resonant frequency is designed by the method of Inductive coupling. The value of $L_{1}$ and $L_{2}$ can be measured by a digital electric bridge. The key challenge for such system is how to achieve maximum electric power of receiver terminal once frequency varies. As shown in figure 3 , it is the curves that matlab code written to calculate and plot the picture of receiving voltage vs. frequency and voltage vs. distance describe voltage vs. frequency distance.

The resonance frequency of $L C$ electric circuit is $f s$. In theory, when $f$ is close to or equals to $f s$, the oscillation of source resonance electric circuit is strongest, the value of resonance current is highest, and the magnetic field intensity $\mathrm{H}$ is also strongest. Inductor $L$ and capacitor $C$ is designed a receiving terminal resonance electric circuit to produce resonance depending on the magnetic field which generated by source terminal resonance electric circuit. The resonance frequency of receiving terminal electric circuit is $f s$, the parameters of $L$ and $C$ need not be in full accord with the source resonance electric circuit. What the receiving terminal resonance electric circuit must need is to ensure $f t=f s$. That is the necessary condition for energy Transmission.

In the work described here, there are two conclusions: This experiment demonstrates wireless electric power transmission with a $L C$ resonant electric circuit; This experiment result shows that the resonant frequency is $18 \mathrm{kHz}$ with $C=100 \mu \mathrm{F}$ at distance $D=3 \mathrm{~mm}$; another resonant frequency is 22 $\mathrm{kHz}$ with $C=470 \mu \mathrm{F}$ at distance $D=4 \mathrm{~mm}$. When the system works at the resonant frequency, $R_{L}$ will receive the maximum electric power and $P_{1}=U^{2} \max / R_{L}=3.1^{2} / 6.9=1.4 \mathrm{~W}, \eta=P_{1} / P_{2}=1.4 \mathrm{~W} / 3.3 \mathrm{~W}=42 \%$.

This electric circuit provides a simple method to achieve resonant coupling between source Tesla coil and receiving Tesla coil.

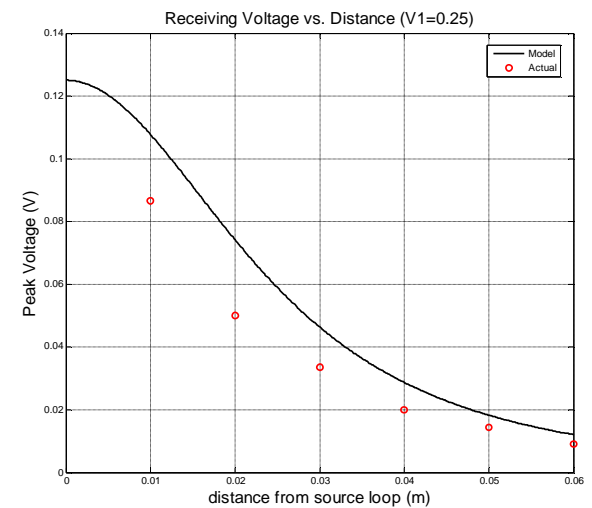

Figure 4. Receiving Voltage(v) vs. Distance(m)

For the experimental setup with a load, the receiving terminal maximum voltage across a resistive bulb placed in the receiving terminal is $3.1 \mathrm{~V}$, corresponding to wireless electric power Transmission delivered electric power of $1.4 \mathrm{~W}$. When the receiver is displaced $10 \mathrm{~mm}$ away from the axis of the transmitting Tesla coil, the voltage drops from $3.1 \mathrm{~V}$ to $0.44 \mathrm{~V}$. In this electric circuit model, electric power disappears by means of electric power dissipation in the resistances. For these parameter values showed in the Figure 4 , much of the electric power leaves in the terminal of the source (electric power dissipation in the resistance $\left.R_{S}\right) .42 \%$ of the electric power is delivered to the load resistance $R_{L}$.

\section{Efficiency of Electric Power Transmission}

The source, operating at frequency of $22 \mathrm{kHz}$, is connected directly across the terminals of the left Tesla coil with diameters of $30 \mathrm{~mm}$, spaced $3 \mathrm{~mm}$ apart. The right Tesla coil is inductively coupled to the left Tesla coil, and is terminated by a capacitor. In the center of the desk is a electric power 
function generator which connected a Tesla coil inductively coupled to another Tesla coil that is connected to a serial capacitor, with the same parameter that is connected to a bulb.

That a small rectification electric circuit connected to $L C$ oscillating electric circuit does deserve to be mentioned. The charging current is about $380 \mathrm{~mA}$. The mobile charger can work well, whose maximum charging voltage is about $4 \mathrm{~V}$.

\section{Summary}

In conclusion, the transferred electric power in the experiment is lower than the total electric power. This is caused by resistance in the Tesla coil and capacitor, because that electrical inductance and capacitor may be a complex electric circuit under high frequency. The maximum electric power transferred by two inductors is at $1.4 \mathrm{~W}$ if the distance separating the inductors is $4 \mathrm{~mm}$. The inductors is diameter $30 \mathrm{~mm}$ with 13 loops per inductor. Based on the experimental result and analysis, it is proved that energy Transmission is completely feasible using this method of magnetic coupling resonance. Nevertheless, there are still a lot of research and experimental work to do to prove the transmission efficiency and Transmission distance. about $42 \%$ of the electric power is delivered to the load that is supplied by a sinusoidal AC source

\section{Acknowledgements}

This work was financially supported by fund project of Civil Aviation Flight University of China (Q2018-170) and (J2015-63). Helicopter multi mission flight training device development, civil aviation innovation and guidance fund projects major projects ( MHRD20130108 ).

\section{References}

[1] A. Kurs, A. Karalis, R.Moffatt, J. D. Joannopoulos, P. Fisher, and M.Soljacic, "Wireless electric power transmission via strongly coupled magnetic resonances," Science, vol. 317, pp. 83-86, Jul. 6, 2007.

[2] Vandevoorde G, Puers R. Wireless energy transmission for standalone systems: a comparison between low and high power applicability. Sensors Actuators. 2001;A 92:305-11.

[3] Benjamin L. Cannon. Inductive coupling As a Potential Method for Wireless Electric power Transmission to Multiple Small Receivers. IEEE Transactions on Electric power Electronics, July 2009, Vol. 24, 1819-1825.

[4] Wang CS, Stielau OH, Covic GA. Load models and their application in the design of loosely coupled inductive power transmission systems. Proceeding of Power System Technology. 2000;12:1053-8.

[5] Ghahary A, Cho BH. Design of transcutaneous energy transmission system using a series resonant converter. IEEE Trans, Power Electron. 1992;7:261-9.

[6] A. Karalis, J. D. Joannopoulos, and M. Soljacic, "Efficient wireless nonradiative mid-range energy transmission," Ann. Phys., vol. 323, no. 1, pp. 34-48, Jan. 2008. 14. Lenaerts B, Puers R. Inductive powering of a freely moving system. Sensors and Actuators. 2005:522-3.

[7] Lenaerts B, Puers R. An inductive power link for a wireless endoscope. Biosensors and Bioelectronics. 2006;6:1-6.

[8] Theodoridis MP, Mollov SV. Distant Energy Transmission for Artificial Human Implants. IEEE Transactions on Biomedical Engineering. November 2005;52:1931-8.

[9] W. C. Brown. The History of Electric power Transmission by Radio Waves. IEEE Trans. Microwave Theory Tech, 1984, Vol. 32, 1230-1242.

[10] K. Finkenzeller, RFIDHandbook: Fundamentals and Applications in Contactless Smart Cards and Identification 2nd ed. New York: Wiley, 2003, ch. 4. 